\title{
Use of Nuclear Medicine Technology for Clinical Molecular Imaging: a Message from the Associate Editor
}

\author{
Byeong-Cheol Ahn ${ }^{1}$
}

Received: 14 March 2016 / Accepted: 28 March 2016 / Published online: 11 April 2016

(C) Korean Society of Nuclear Medicine 2016

Molecular imaging is defined as the visual representation, characterization, and quantification of biological processes at the cellular and subcellular levels within intact living organisms; this can be achieved by various imaging technologies such as nuclear imaging, optical imaging, magnetic resonance imaging (MRI), ultrasound imaging, and computed tomography (CT) [1]. Although these modalities are actively used for sensing molecular and cellular processes in preclinical studies, the majority of imaging techniques remain unapplied in the clinical field due to various unresolved issues, such as safety, poor tissue penetration of the imaging signal, and limited sensitivity. So far, nuclear or fusion imaging modalities with the nuclear medicine component are the actively used molecular imaging modalities in the clinical field [1-3].

Nuclear imaging has many fundamental principles as molecular imaging. Nuclear medicine imaging has provided molecular information since the early years of imaging technology. In 1951, Dr. Benedict Cassen successfully imaged the thyroid gland with his rectilinear scanner; his imaging principle is still widely used in daily clinical practices to elucidate iodine avidity of benign and malignant thyroid follicular cells. F-18 FDG PET imaging is probably the most well-known clinical molecular imaging technique that can be used to visualize activity of glucose metabolism. The imaging technique is widely used in the oncologic arena as the

Byeong-Cheol Ahn

abc2000@knu.ac.kr

1 Department of Nuclear Medicine, Kyungpook National University School of Medicine and Hospital, 50, Samduk 2-ga, Jung Gu, Daegu, Republic of Korea 700-721 majority of tumors utilize glucose more actively than normal tissues. High avidity of radioiodine in thyroid follicular cells functions as the tracer for thyroid diseases, while high glucose avidity of tumor functions as F-18 FDG for oncologic disorders. The elucidation of pathophysiology of certain diseases lead to the development of new diagnostic tracers, which in turn shed more light on the disease pathophysiology. This virtuous cycle would aid in uncovering diseases at an early phase and might increase the chance of complete cure by identifying appropriate molecular signatures of diseases.

Nuclear molecular imaging was and still is the leading molecular imaging technology. Although nuclear molecular imaging has distinct advantages over other imaging modalities, it has its own limitations, such as low spatial/temporal resolution and high background signal. Multimodal fusion imaging might be a good strategy to overcome the disadvantages of molecular imaging modalities; PET/CT or PET/MRI is already widely installed and actively used in clinics to provide the best molecular imaging service [4].

In fact, clinical molecular imaging already plays indispensable roles in optimal patient care, and its diagnostic and theranostic potentials are increasingly recognized through better understanding of disease pathophysiology in combination with rapid advancements of electronic and biologic technologies. Constant contributions of nuclear medicine physicians and nuclear/biological scientists will retain nuclear molecular imaging as the leading clinical imaging technology to visualize hidden molecular events in human studies and link successful ideas from basic preclinical environment to clinical translation [5]. Nuclear Medicine and Molecular Imaging would be the platform for the advancement of nuclear molecular imaging and provide cutting edge 
information that relates to clinical molecular imaging using nuclear medicine technology.

Acknowledgments This work was supported by grants from the Korea Health Technology R\&D Project through the Korea Health Industry Development Institute (KHIDI), funded by the Ministry of Health \& Welfare, Republic of Korea (grant number : HI15C0001) and the National Research Foundation of Korea (NRF) grant funded by the Korea government (MSIP) (No. NRF-2015M2A2A7A01045177)

\section{Compliance with Ethical Standards}

Conflict of Interest Byeong-Cheol Ahn declared no conflict of interest.

\section{References}

1. Ahn BC. Sodium iodide symporter for nuclear molecular imaging and gene therapy: from bedside to bench and back. Theranostics. 2012;2:392-402.

2. Ahn BC. Applications of molecular imaging in drug discovery and development process. Curr Pharm Biotechnol. 2011;12:459-68.

3. Kim JE, Kalimuthu S, Ahn BC. In vivo cell tracking with bioluminescence imaging. Nucl Med Mol Imaging. 2015;49:3-10.

4. Miller JC, Thrall JH. Clinical molecular imaging. J Am Coll Radiol. 2004;1(Suppl):4-23.

5. van Es SC, Venema CM, Glaudemans AW, et al. Translation of new molecular imaging approaches to the clinical setting: bridging the gap to implementation. J Nucl Med. 2016;57:96S-104S. 